\title{
RESOLUÇÃO CIRÚRGICA DE PERIODONTITE APICAL CRÔNICA: RELATO DE CASO
}

\section{SURGICAL RESOLUTION OF CHRONIC APICAL PERIODONTITIS: CASE REPORT}

\author{
Renato Piai Pereira* \\ João Milton Rocha Gusmão* \\ Adriano Monteiro d' Almeida Monteiro**. \\ Alex Correia Vieira**** \\ Juliano Fernandes Sassi ${ }^{* \cdots \cdots}$ \\ Luiz Roberto Mendes da Silva*****
}

\begin{abstract}
RESUMO
A cirurgia paraendodôntica é um conjunto de procedimentos com o objetivo básico de tratar lesões perirradiculares decorrentes de complicações do tratamento endodôntico ou seu insucesso. O objetivo deste trabalho é apresentar um caso clínico de cirurgia paraendodôntica em um incisivo lateral superior. O tratamento da lesão perirradicular foi realizado através da curetagem, apicectomia, retropreparo com inserto de ultrassom e retro-obturação com MTA. O exame histopatológico confirmou o diagnóstico de granuloma periapical. O controle de um ano demonstrou a neoformação óssea e ausência de sintomatologia.
\end{abstract}

Descritores: Endodontia • Apicectomia • Obturação retrógrada.

\section{ABSTRACT}

Apical surgery is a set of procedures with the basic aim of treating complications arising from lesions of endodontic treatment or its failure. The objective of this study is to present a clinical case of apical surgery in a maxillary lateral incisor. The treatment of lesion was performed by curettage, apicectomy, root-end preparation with ultrasonic unit and root-end filling with MTA. The biopsy confirmed the diagnosis of apical granuloma. The control for a year showed new bone formation and absence of symptoms.

Descriptors: Endodontics • Apicoectomy • Retrograde obturation.

\footnotetext{
* Universidade Estadual do Sudoeste da Bahia - Mestre em Endodontia; Professor Assistente em Endodontia; rppiai@bol.com.br

** Mestre em Prótese Dentária; Professor Assistente em Prótese; joao.milton@ig.com.br

*** Doutor em Imunologia; Professor Assistente em Periodontia; amdmonteiro@yahoo.com.br

**** Mestre em Odontologia Clínica; Professor Assistente em Dentística; lekovieira@ hotmail.com

***** Mestre em Dentística Restauradora; Professor Assistente em Dentística; jfsassi@gmail.com

****** Especialista em Endodontia; Professor Auxiliar em Endodontia; robertomendes@uesb.edu.br
} 
PEREIRA RP

GUSMÃO JMR

MONTEIRO AMA

VIEIRA AC

SASSI JF

SILVA LRM

RESOLUÇÃO

CIRÚRGICA DE

PERIODONTITE

APICAL CRÔNICA:

RELATO DE CASO

REV, ODONTOL.

UNIV, CID, SÃO PAULO

2013; 25(1): 77 -

82 , JAN-ABR

\section{IN T RO DUÇÃ 0}

Segundo Ng et al. ${ }^{1}$ (2011), o insucesso da terapia endodôntica pode estar relacionado a diversos fatores como doença periodontal, presença de lesão periapical prévia, fraturas radiculares, perfurações, complicações anatômicas, problemas relacionados a procedimentos restauradores, dentre outros.

A manutenção da doença periapical está relacionada à presença de microrganismos no canal radicular ou até mesmo na superfície externa radicular ${ }^{2,3}$. Algumas lesões persistentes após o tratamento endodôntico podem ocorrer pela presença de microrganismos fora do canal radicular, junto aos tecidos periapicais, em locais inacessíveis à terapia endodôntica não cirúrgica. Bactérias, sobretudo anaeróbias estritas, podem deixar o canal radicular infectado e penetrar nos tecidos perirradiculares, mantendo o processo infeccioso, pois podem estruturar-se em forma de biofilme, evadindo-se dos sistemas de defesa do hospedeiro ${ }^{4,5}$. Toledo ${ }^{6}$ (2011) demonstrou um caso de lesão periapical, não resolvido após tratamento endodôntico convencional, necessitando de intervenção cirúrgica. Após o tratamento cirúrgico foi observada a presença de depósitos de cálculo na superfície radicular do fragmento apical removido.

O tratamento cirúrgico somente está indicado quando o convencional não for possível ou falhar. Nos últimos anos, o surgimento intenso de novas técnicas e materiais proporcionou uma melhoria na qualidade dos procedimentos endodônticos, reduzindo significativamente a necessidade de intervenções cirúrgicas na região periapical; assim, o tratamento endodôntico cirúrgico está indicado em limitado número de casos de patologias perirradiculares persistentes ${ }^{7}$.

A cirurgia paraendodôntica é um conjunto de procedimentos com o objetivo básico de tratar lesões perirradiculares decorrentes de complicações do tratamento endodôntico ou seu insucesso, de acor$\mathrm{do}^{8}$. O objetivo deste trabalho consiste na apresentação de um caso clínico de cirurgia paraendodôntica.

\section{DESCRIÇÃO E DISCUSSÃO DO CASO} CLÍNICO

O paciente, gênero feminino, 50 anos, compareceu ao módulo de Odontologia da Universidade Estadual do Sudoeste da Bahia (UESB), na disciplina de Clínica Integrada VI, para avaliação e tratamento de um incisivo lateral superior (22), portador de prótese coronária e retentor intrarradicular, com diagnóstico clínico sugestivo de periodontite apical crônica.

$\mathrm{Na}$ análise clínica da unidade 22, foi verificada a qualidade da adaptação da prótese unitária fixa. Durante os testes semiotécnicos observou-se presença de dor à palpação apical. O exame periodontal demonstrou ausência de sangramento e distâncias biológicas dentro dos padrões de normalidade.

$\mathrm{Na}$ avaliação da radiografia periapical (Figura 1) observa-se imagem sugestiva de rarefação óssea periapical, tratamento endodôntico dentro dos limites aceitáveis e presença de retentor intrarradicular, radiograficamente bem adaptado, apresentando comprimento e espessura adequados ao remanescente radicular. Com base na história, exame clínico e exame radiográfico, decidiu-se pelo planejamento e intervenção cirúrgica. A paciente optou pelo plano de tratamento proposto.

Com a paciente anestesiada, inicio-se a manobra cirúrgica, com incisão tipo Newmann, intrassulcular, por vestibular, estendendo-se da mesial do 11 à distal do 23, com bisturi e lâmina Bard Parker $\mathrm{n}^{\circ}$ 15. Sequencialmente fora realizada uma segunda incisão relaxante na distal da unidade 23, preservando-se a papila distal da referida unidade, estendendo-se até a mucosa de revestimento alveolar (Figura 2).

Após a divulsão total do retalho e exposição da região periapical do 22 , realizou-se a ostectomia com broca esférica cirúrgica (Komet Brazil, Santo André, SP, Brasil), sob irrigação com solução salina (Figura 3), para expor o tecido patológico e o ápice radicular. O tecido mole foi cuidadosamente curetado e fixado em formol a $10 \%$, para ser encaminhado ao exame histopatológico. Com uma broca multilaminada (Komet Brazil, Santo André, SP, Brasil) foi realizado um corte de aproxi- 


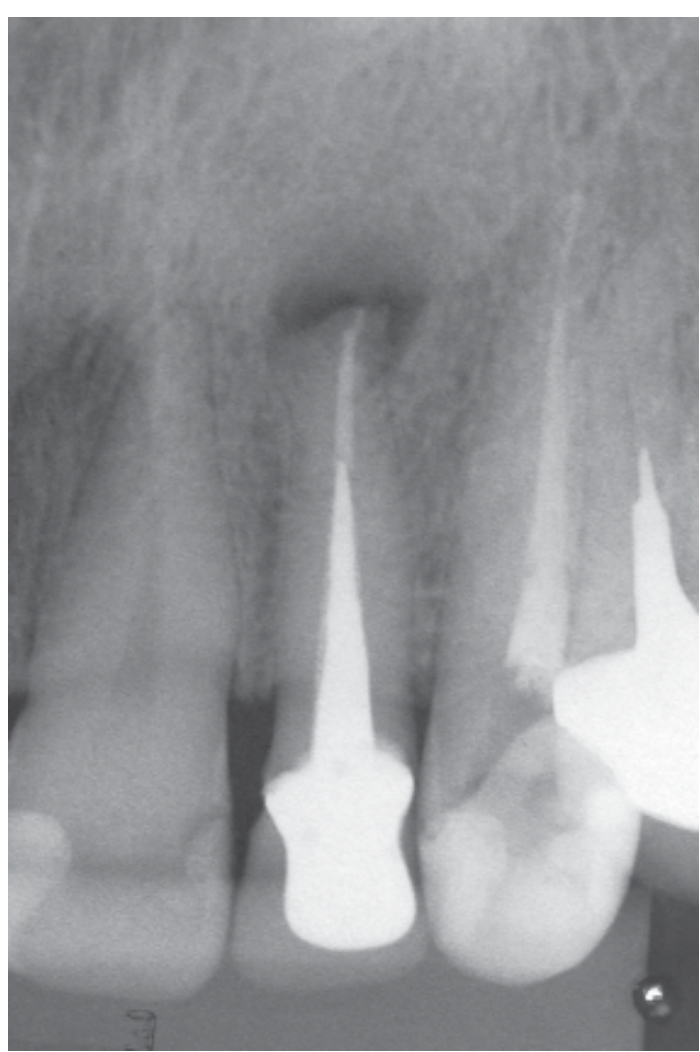

Figura 1 - Radiografia do estudo

madamente $3 \mathrm{~mm}$ da porção apical (Figura 4).

O preparo da retrocavidade foi realizado com uma ponta de ultrassom TRA21
(Trinks ${ }^{\circledR}$, São Paulo, SP, Brasil), sob irrigação com solução salina (Figura 5). Após a secagem com pontas de papel absorventes, a cavidade foi preenchida com MTA branco (Angelus ${ }^{\circledR}$, Londrina, PR, Brasil), inserido com o auxílio de um pequeno porta-amálgama e condensadores (Figuras 6). Após a remoção do excesso de material e limpeza da loja cirúrgica, o retalho foi reposicionado, suturado e realizada a radiografia pós-operatória (Figura 7).

O exame histopatológico confirmou o diagnóstico de granuloma periapical. O controle radiográfico de 01 ano sugeriu a neoformação óssea (Figura 8).

Um correto diagnóstico e planejamento são fundamentais para o sucesso da terapia proposta. A escolha pelo retratamento cirúrgico no presente caso clínico foi a persistência da lesão periapical após a terapia endodôntica. Segundo Arens et al. ${ }^{8}$ (1998), em elementos dentários portadores de retentor intrarradicular, o retratamento cirúrgico é uma alternativa. A qualidade do tratamento endodôntico e restaurador foi avaliada, onde os limites apicais de obturação, o comprimento e espessura do retentor estavam dentro de padrões aceitáveis, bem como uma boa adaptação exibida pela prótese fixa, haja
PEREIRA RP GUSMÃO JMR

Monteiro ama

VIEIRA AC

SASSI JF

SILVA LRM

RESOLUÇÃO

CIRÚRGICA DE

PERIODONTITE

APICAL CRONNICA:

RELATO DE CASO

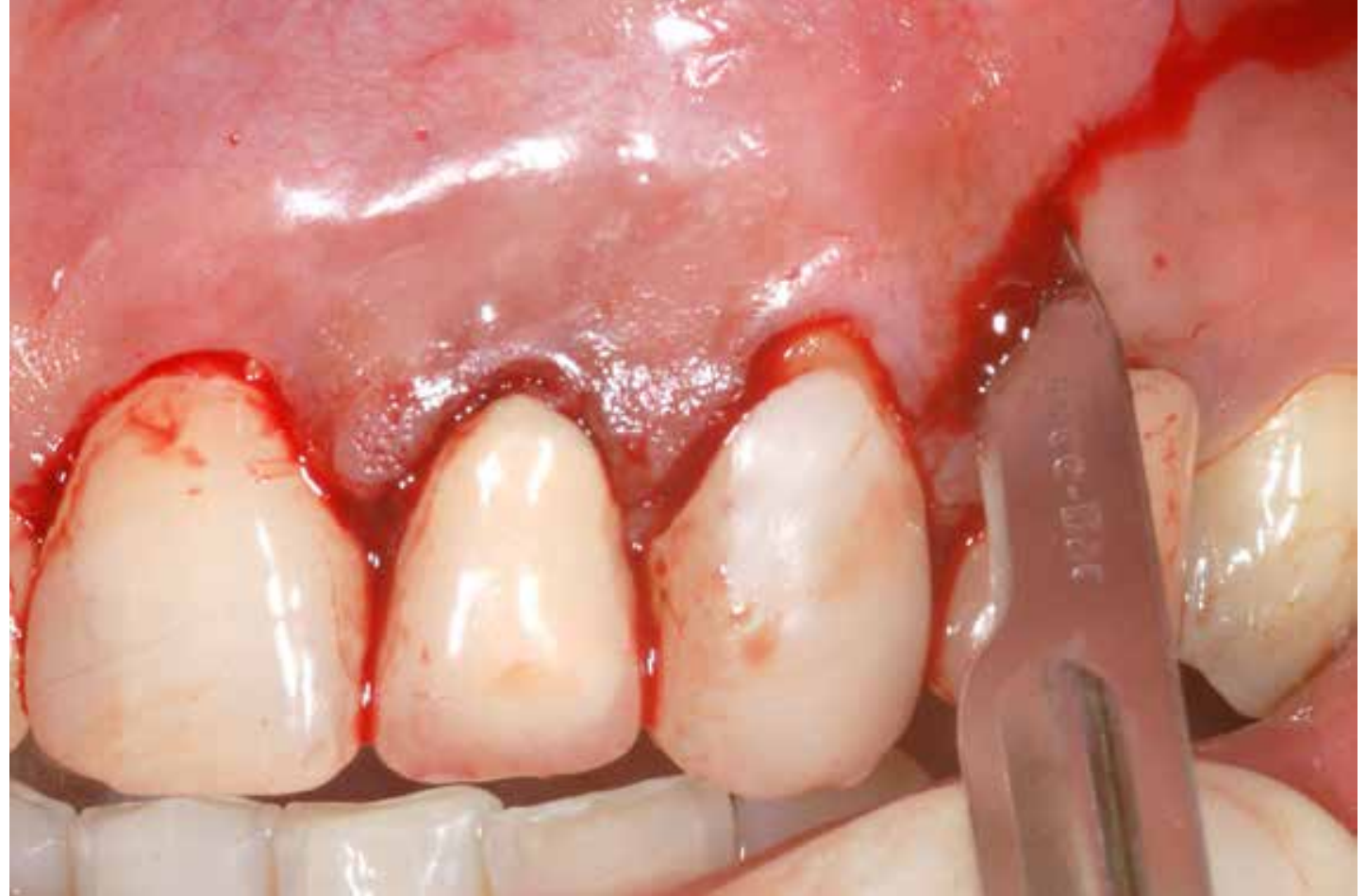

Figura 2 - Incisão
REV, ODONTOL.

UNIV, CID, SÃo

PAULO

2013; 25(1): 77 -

82, JAN-ABR 


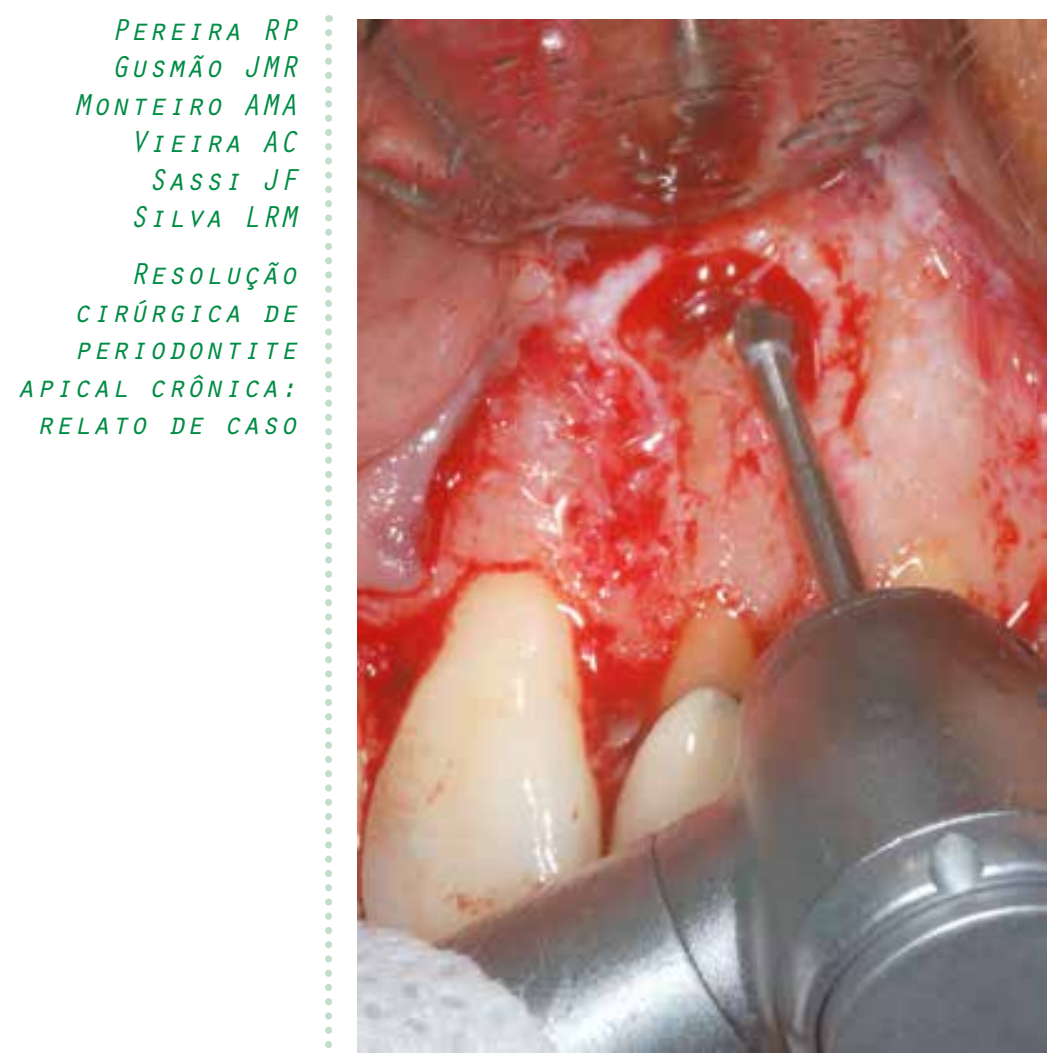

Figura 3 - Ostectomia

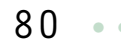

REV, ODONTOL.

UNIV, CID, SÃO

PAULO

2013; 25(1): 77 -

82 , JAN-ABR

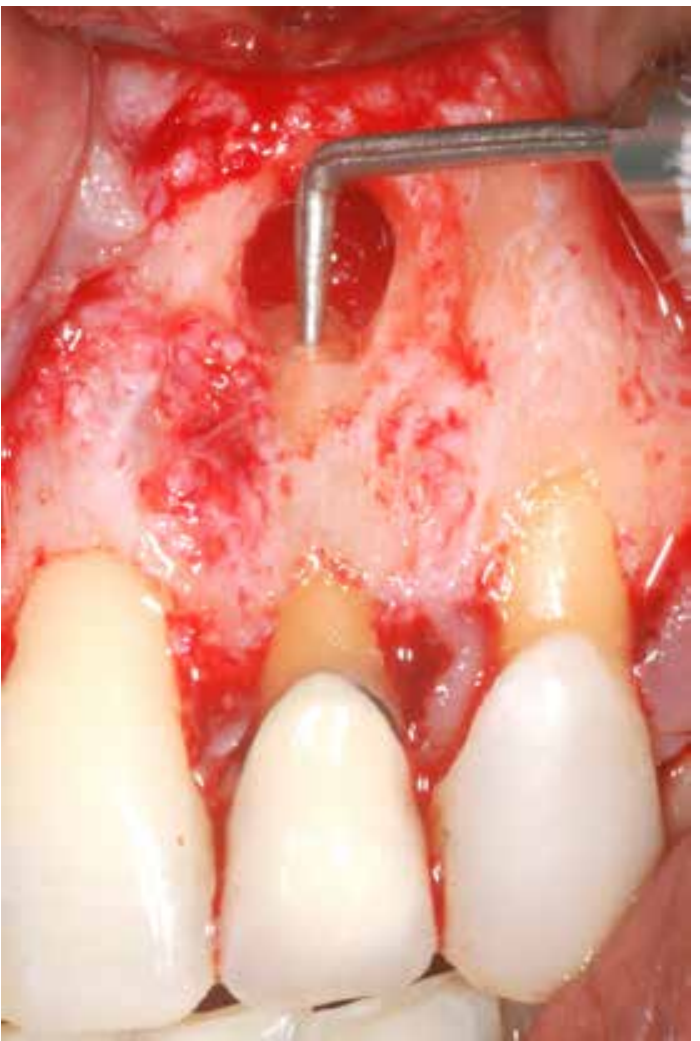

Figura 5 - Preparo de retrocavidade

vista que a microinfiltração coronária é apontada como uma das causas de insucesso em Endodontia ${ }^{9}$.

O êxito em cirurgia paraendodôntica depende de passos importantes como o

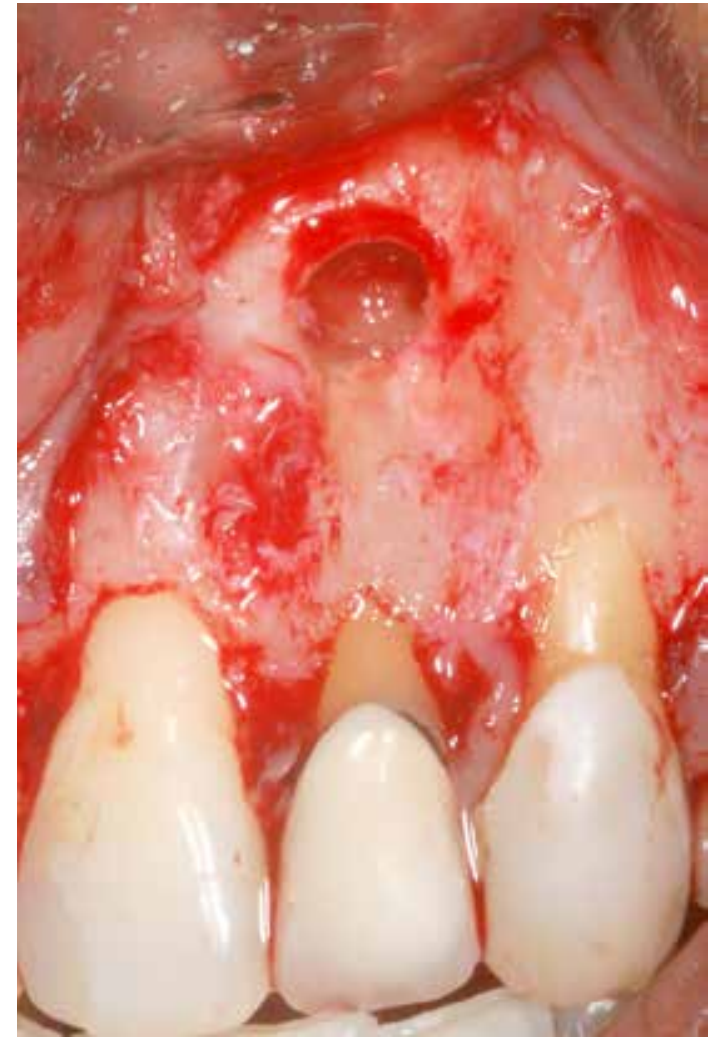

Figura 4 - Apicectomia

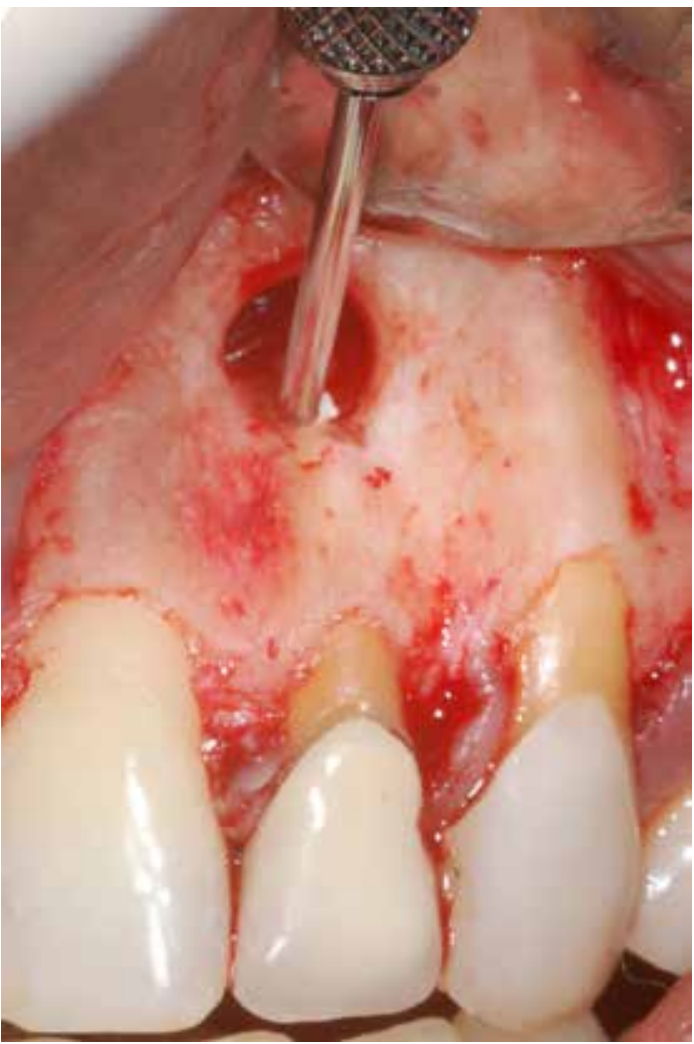

Figura 6 - Inserção do MTA

acesso cirúrgico, o preparo apical e a escoIha do material retro-obturador. A cirurgia paraendodôntica fornece a oportunidade de remover a lesão periapical e porção apical da raiz, região de difícil acesso às 


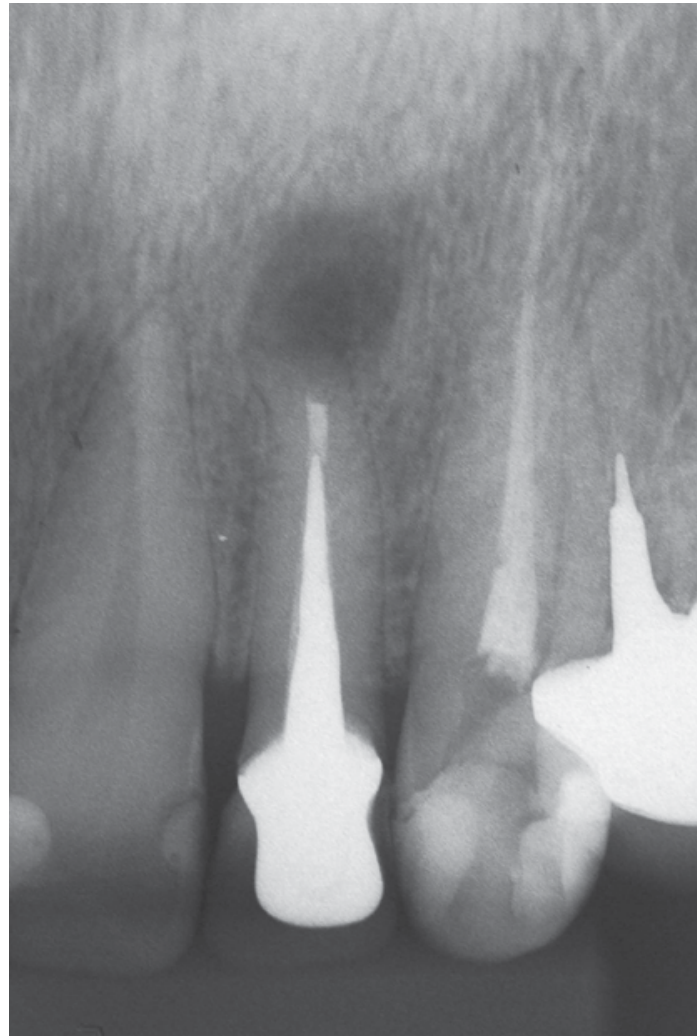

Figura 6 - Radiografia pós-operatória

substâncias químicas e com as maiores variações anatômicas do canal radicular ${ }^{10}$. A remoção do terço apical também possibilita a eliminação da infecção extrarradicular, que pode manter a lesão periapical após tratamento endodôntico ${ }^{2,3}$.

O retropreparo com pontas ultrassônicas exibe inúmeras vantagens quando comparado com brocas. Permite uma osteotomia menor, para o acesso cirúrgico, por causa do pequeno tamanho das pontas, preparo cavitário mais conservador e paralelo ao canal radicular, assim realizando-se com um debridamento mais eficiente, principalmente em áreas de istmos. Relatam Tsesis et al. ${ }^{11}$ (2006) e Tavares et al. ${ }^{12}$ (2011) que o remanescente dentário é mais preservado, uma vez que o corte apical pode ser menos inclinado, com menor exposição de túbulos dentinários.

A retro-obturação apical tem como objetivo vedar o sistema de canais, impedindo que microrganismos que ainda persistam no interior do sistema de canais radiculares e seus subprodutos alcancem os tecidos periapicais ${ }^{13}$. Para tal, a escolha

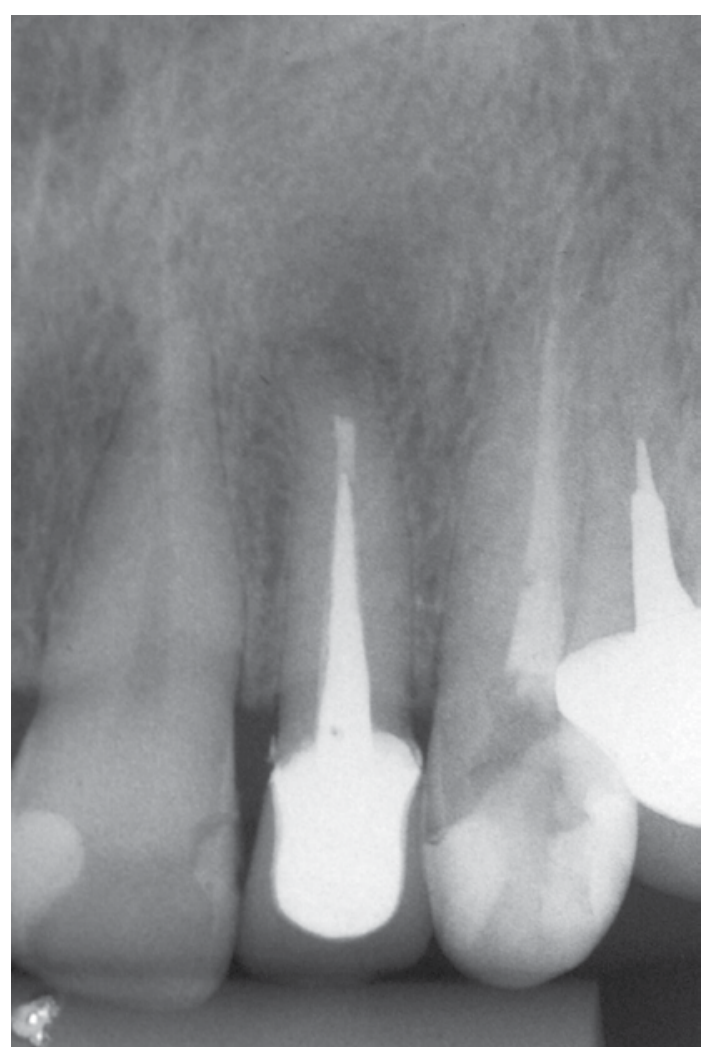

Figura 6 - Controle radiografico de 01 ano

do material tem grande importância, pois pode influenciar no sucesso clínico a longo prazo. Neste relato de caso, o material escolhido foi o MTA. O material empregado deve ser biocompatível e apresentar boas propriedades biológicas, físicas e químicas. O MTA (Agregado Trióxido Mineral) parece apresentar boas características e seus resultados positivos sustentam seu emprego ${ }^{14,15}$. Segundo Holland et al. ${ }^{16}$ (2001), histologicamente observa-se ausência de inflamação no ligamento periodontal, além da deposição de cemento sobre o material, na maioria dos casos selados com o MTA.

\section{CONCLUSÃO}

Diante dos resultados obtidos, conclui-se que a cirurgia paraendodôntica, através do uso das pontas ultrassônicas para preparo da retrocavidade e da retro-obturação com MTA, foi apropriada para a resolução do caso em questão, o que ficou comprovado clínica e radiograficamente por meio da ausência de sintomatologia e da neoformação óssea.
PEREIRA RP GUSMÃO JMR

MONTEIRO AMA

VIEIRA AC

SASSI JF

SILVA LRM

RESOLUÇÃO

CIRÚRGICA DE

PERIODONTITE

APICAL CRONICA:

RELATO DE CASO

REV, ODONTOL.

Univ. Cid. São

PAulo

$2013 ; 25(1): 77$ -

82 , JAN-ABR 
PEREIRA RP

GUSMÃO JMR :

MONTEIRO AMA

VIEIRA AC

SASSI JF

SILVA LRM

RESOLUÇÃO

CIRÚRGICA DE

PERIODONTITE

APICAL CRONNICA:

RELATO DE CASO
REV, ODONTOL.

UNIV, CID. SÃO PAULO

2013; 25(1): 77 82, JAN-ABR

\section{REFERÊNCIAS}

1. Ng YL, Mann V Gulabivala K. A prospective study of the factors affecting outcomes of nonsurgical root canal treatment: part 1: periapical health. Int Endod J 2011;44(7):583-609.

2. Leonardo MR, Rossi MA, Silva LA, Ito IY, Bonifácio KC. EM evaluation of bacterial biofilm and microorganisms on the apical external root surface of human teeth. $J$ Endod 2002;28(12):815-8.

3. Nair PN. On the causes of persistent apical periodontitis: a review. Int Endod J 2006;39(4):249-81.

4. Byström A, Happonen RP, Sjogren U, Sundqvist G. Healing of periapical lesions of pulpless teeth after endodontic treatment with controlled asepsis. Endod Dent Traumatol 1987;3(2):58-63.

5. Sjögren U, Hagglund B, Sundqvist G, Wing K. Factors affecting the long-term results of endodontic treatment. J Endod 1990;16(10):498-504.

6. Toledo JRG. Resolução cirúrgica de periodontite apical em dente com formação cálculo via trajeto fistuloso. Rev Assoc Paul Cir Dent 2011;65(1):42-7.

7. Cohn SA. When all else fails.... Aust Endod J 1998;24(3):128-9.

8. Arens DE, Torabinejad M, Chivian N, Rubinstein R. Pratical lessons in endodontic surgery. Illinois: Quintessence; 1998.

9. Ng YL, Mann V, Gulabivala K. A prospective study of the factors affecting outcomes of non-surgical root canal treatment: part 2: tooth survival. Int Endod J 2011;44(7):61025.

10. Oliveira MAVC, Soares J, Azevedo KCM, Biffi JCG, Quirino LC, Faria RA. Cirurgia paraendodôntica como complemento do tratamento endodôntico: relato de caso clínico. Endodontics 2011;1(2):70-4.

11. Tsesis I, Rosen E, Schwartz-Arad D, Fuss Z. Retrospective evaluation of surgical endodontic treatment: traditional versus modern technique. J Endod 2006;32(5):412-6.

12. Tavares WLF, Lopes RCP, Henriques LCF, Menezes GB, Ribeiro Sobrinho AP. MeIhora dos resultados clínicos em casos complexos através da microcirurgia endodôntica moderna: uma série de casos. Endodontics 2011;1(2):81-8.

13. Song M, Shin SJ, Kim E. Outcomes of endodontic micro-resurgery: a prospective clinical study. J Endod 2011;37(3):316-20.

14. Chong BS, Pitt Ford TR, Hudson MB. A prospective clinical study of Mineral Trioxide Aggregate and IRM when used as root-end filling materials in endodontic surgery. Int Endod J 2003;36(8):520-6.

15. Torabinejad M, Parirokh M. Mineral trioxide aggregate: a comprehensive literature review-part II: leakage and biocompatibility investigations. J Endod 2010;36(2):190202.

16. Holland R, Filho JA, de Souza V, Nery MJ, Bernabé PF, Junior ED. Mineral trioxide aggregate repair of lateral root perforations. J Endod 2001;27(4):281-4.

Recebido em: 24/11/2011

Aceito em: 09/04/2012 